
\title{
25 Research Soure \\ The Feasibility of Combining Brain Radiation and Fingolimod in Healthy Rodents and in Humans With Newly Diagnosed Glioblastoma
}

Stuart A. Grossman ( $\nabla$ grossman@jhmi.edu )

Johns Hopkins Oncology Center https://orcid.org/0000-0002-3726-7679

Anna Piotrowski

Memorial Sloan Kettering Cancer Center

Dvone Jackson

University of Pennsylvania

Thomas Nirschl

Johns Hopkins: Johns Hopkins University

Esteban Velarde

Johns Hopkins: Johns Hopkins University

John Wong

Johns Hopkins: Johns Hopkins University

Charles Drake

Columbia University Irving Medical Center

Xiaobu Ye

Johns Hopkins: Johns Hopkins University

\section{Research Article}

Keywords: Radiation, Lymphopenia, Animal Model, Fingolimod, Glioblastoma

Posted Date: March 2nd, 2021

DOI: https://doi.org/10.21203/rs.3.rs-251903/v1

License: (c) (i) This work is licensed under a Creative Commons Attribution 4.0 International License.

Read Full License 


\section{Abstract}

PURPOSE: Severe and long-lasting lymphopenia occurs in $40 \%$ of patients with malignant gliomas and is associated with inferior survival. Inadvertent irradiation of circulating lymphocytes is a major contributor to this lymphopenia. Fingolimod causes striking but reversible lymphopenia by sequestering circulating lymphocytes in lymphoid tissues. Fingolimod could potentially reduce iatrogenic immunosuppression by decreasing the number of lymphocytes in circulation during radiation. However, combining radiation with fingolimod should result in severe lymphopenia. These pilot studies were conducted to determine the safety of this approach in mice and humans.

METHODS: Ten BALB/c mice received focal brain irradiation (4Gy $\times 10$ fractions). Half of the mice received intraperitoneal fingolimod $(3 \mathrm{mg} / \mathrm{kg}$ ) before and during the two weeks of radiation. Five patients with newly diagnosed glioblastoma were given fingolimod one week prior to and during the six weeks of concurrent radiation and temozolomide.

RESULTS: Mice treated with fingolimod and radiation had more severe lymphopenia than those treated with radiation alone. However, lymphocyte counts and weight loss recovered similarly in both treatment cohorts and no added toxicities were noted. Humans receiving fingolimod developed severe lymphopenia which deepened when radiation and temozolomide were initiated. This was well tolerated and no grade III-IV opportunistic infections were noted.

CONCLUSIONS: These pilot studies demonstrate the feasibility and safety of combining fingolimod with radiation in mice and humans. This novel approach to reducing the number of circulating lymphocytes exposed to radiation deserves further study given the importance of the host immune system on cancer survival and response to immunologic interventions.

\section{Background}

It has long been known that immunosuppression is associated with an increased incidence of cancer and with inferior outcomes in patients with cancer. More recently, studies have documented that approximately $40 \%$ of patients with a wide variety of cancers who receive radiation and chemotherapy develop grade III-V lymphopenia which is characterized by a total lymphocyte counts $<500 / \mu \mathrm{l}$ and CD 4 counts $<200 / \mu \mathrm{l}$.[1-6] This severe treatment-related lymphopenia (TRL) peaks at the end of radiation, persists for over one year, and is associated with early recurrence and death from cancer on multivariate analyses. These observations were initially described in patients with glioblastoma treated with standard radiation, temozolomide, and clinically indicated doses of glucocorticoids.[1]

The precise mechanisms underlying this lymphopenia have not been fully defined. However, currently available studies have demonstrated that the degree of treatment-related lymphopenia is independent of tumor histology, steroid administration, or the type of chemotherapy administered.[6] There is compelling evidence that this iatrogenic immune injury is related to the inadvertent radiation of blood circulating through the radiation field.[7-8] Prior animal studies, including work performed by our group, and one 
clinical study have shown that extracorporeal radiation of circulating blood results in deep and prolonged lymphopenia.[9-11] In addition, a mathematical model has been constructed to calculate the dose of radiation delivered to circulating lymphocytes.[8] This demonstrates that larger treatment volumes, lower radiation dose rates, and a higher number of radiation fractions increase the toxicity to lymphocytes which are the most radiation sensitive cells in the body. This model has also been used to estimate the dose of radiation administered to circulating blood in patients with glioblastoma. Although standard radiation fields for patients with glioblastoma do not include bone marrow or lymphoid organs, after 30 radiation fractions virtually the entire circulating blood pool has received lymphotoxic doses of radiation. [8]

There are several approaches that should reduce radiation exposure to circulating lymphocytes, such as reductions in the treatment volume, the number of administered fractions, and the time that the radiation beam is on per fraction.[12-16] Another promising approach to reducing this inadvertent toxicity to the immune system in patients with cancer is to encourage lymphocytes to temporarily leave the circulation while radiation is being administered. Fingolimod is an FDA approved sphingosine 1 phosphate (S1P) agonist used in the treatment of multiple sclerosis.[17] This agent interferes with the normal circulation of lymphocytes by impeding their exit from lymphoid organs that results in a $70 \%$ reduction in the number of circulating lymphocytes. Once fingolimod is discontinued the numbers of circulating lymphocytes return towards normal. In practice, starting fingolimod one week prior to radiation would allow time for circulating lymphocytes to fall by $70 \%$. This would result in fewer circulating immune cells passing through the radiation beam. Upon completion of the radiation course, discontinuing fingolimod would permit the recovery of circulating lymphocyte counts. However, there are potential risks to adding fingolimod to standard radiation. Given that standard radiation and temozolomide results in severe lymphopenia in $40 \%$ of patients with glioblastoma and that fingolimod reduces circulating lymphocyte counts by $70 \%$ in patients with multiple sclerosis, combining therapies would be expected to result in an especially severe drop in lymphocyte counts which, in turn could lead to the development of serious opportunistic infections or a significant delay in lymphocyte count recovery.

In this manuscript, we report the first pre-clinical study combining focused cranial irradiation with fingolimod using a recently described animal model of treatment-induced lymphopenia.[9] In this murine model, circulating lymphocyte counts fall sharply after radiation but, unlike treatment-related lymphopenia in humans, their lymphocyte counts rapidly and spontaneously recover.[11] This murine model was used to determine if: 1) there were obvious infectious complications resulting from severe lymphopenia caused by focused cranial irradiation and fingolimod dual therapy and 2) if fingolimod delayed the spontaneous recovery of lymphocytes following completion of radiation in rodents. In addition, we are reporting the first human safety data using fingolimod with radiation and temozolomide in patients with newly diagnosed high grade glioma. These two pilot studies provide convincing data to suggest that this novel approach to ameliorate radiation-related lymphopenia can be safely studied in prospective randomized studies in patients receiving standard radiation therapy. 


\section{Materials And Methods - Murine Study}

The murine model utilized in this study is described in detail in the literature.[9] The animal species and radiation doses and durations used in this study were selected from the original model development paper. This study was approved by the Animal Care and Use Committee of the Johns Hopkins University School of Medicine.

Animals: Ten male BALB/c mice, 7-12 weeks old were purchased from The Jackson Laboratory.

Radiation: A total of $40 \mathrm{~Gy}$ (4 Gy in 10 fractions) was administered over two weeks to the brains of mice using the small animal radiation research platform (SARRP) (Xstrahl, Inc). Animals were anesthetized before treatment using isoflurane then immobilized on the rotary bed. The SARRP is equipped with cone beam computed tomography (CBCT) which was used for an image guided and highly conformal radiation delivery to the murine brain. An AP (anterior to posterior) beam of $10 \times 10 \mathrm{~mm}$ at a depth of 5 $\mathrm{mm}$ (345 SSD) was used. The energy of this beam was $220 \mathrm{kVp}$ (voltage) and $13 \mathrm{~mA}$ (current). The system's accuracy of the beam position is $0.2 \mathrm{~mm}$.

Fingolimod: The control group of animals $(\mathrm{N}=5)$ received focal brain radiation alone while the experimental group ( $\mathrm{N}=5$ ) received radiation plus fingolimod at dose $3 \mathrm{mg} / \mathrm{kg}$. Fingolimod was provided by the laboratory of Dr. Peter Calabresi for the animal studies. This drug has been extensively studied in murine models of multiple sclerosis. The dose selected for this study was known to be efficacious in reducing lymphocyte counts in mice. Three $\mathrm{mg} / \mathrm{kg}$ were injected intraperitoneally daily for 4 days prior to the start of radiation and throughout the 2-week course of radiation.

Tolerability: Tolerability was assessed by daily observations of body mass, and a composite scoring system taking into account posture, eye appearance, and activity level. ${ }^{18}$ Additionally, blood tests were obtained every 4 days (total lymphocyte count [TLC], absolute neutrophil count, hematocrit, platelet count).

\section{Results - Murine Study}

Serial observations of posture, eye appearance, and overall activity levels were very similar in the control (radiation alone) and the experimental (radiation with fingolimod) groups (data not shown). As expected, animal weights decreased approximately $30 \%$ from baseline when compared to one-week post radiation (day 21) and then returned to baseline by day 40 . There was no difference in the weight curves by treatment arms (Figure 1a). Similarly, comparisons of the absolute neutrophil counts, hematocrits, and platelet counts were unaffected by the addition of fingolimod to cranial irradiation (Figures 1b, 1c, 1d). However, treatment with fingolimod dramatically reduced lymphocyte counts from a baseline of 4,800 cells/ $\mu$ l to $660 \mathrm{cells} / \mu \mathrm{l}$ days before radiation was initiated (Figure 1e). Furthermore, the circulating lymphocyte count remained more than $50 \%$ lower in the experimental group, when compared to the control group, during the entire course of radiation. Fingolimod and radiation were discontinued on day 16 , and by day 28 the lymphocyte counts in both groups were similar. Importantly, there were no 
unexpected deaths, infections, or other serious toxicities noted in the animals treated with radiation plus fingolimod.

\section{Materials And Methods - Human Study}

Study design: This IRB approved five patient open-label, single arm, single institution pilot safety study was designed to determine if patients receiving standard radiation, temozolomide and an FDA approved dose of fingolimod would develop severe opportunistic infections or other complications that would prohibit further evaluation of this novel treatment approach to radiation-induced lymphopenia. The fingolimod was initiated one week prior to beginning the radiation $(0.5 \mathrm{mg}$ orally daily for two weeks followed by $0.5 \mathrm{mg}$ on Monday, Wednesday, and Friday) and was discontinued upon the completion of radiation therapy. Complete blood counts were obtained weekly, CD4 counts were obtained monthly, and the patients were monitored closely for evidence of infection or toxicity. For the purposes of this study, dose limiting toxicities were defined as Grade III-IV infections ( $\mathrm{NIH} / \mathrm{NCl}$ Common Terminology Criteria for Adverse Events) attributable to fingolimod-induced lymphopenia within four months of starting fingolimod or death from any cause attributable to fingolimod. If one of the five accrued and evaluable patients developed a Grade III-IV infection, this would signify that fingolimod with radiation and temozolomide is not safe. The secondary objective was to obtain preliminary information regarding the depth and recovery of radiation-related lymphopenia with this combination therapy. This safety study was not blinded or placebo controlled. A study schema with the patient population, study designs, objectives, and endpoints is provided in Figure 2.

Eligibility requirements: Eligible patients were required to meet the following inclusion criteria: 1) newly diagnosed grade III or IV astrocytomas who were scheduled to begin standard radiation and temozolomide, 2) age $\geq 18$ years, 3) Karnofsky performance status $\geq 60,4$ ) normal bone marrow function, 5) baseline total lymphocyte count of greater than 1000,6) willing to use appropriate contraception, and 7) able to provide informed consent. Exclusion criteria were: 1) prior radiation therapy, systemic chemotherapy, immunotherapy or other non-surgical therapy for their brain tumor, 2) recent (within six months) myocardial infarction, unstable angina, stroke, transient ischemic attack, decompensated heart failure requiring hospitalization, or Class III/IV heart failure. 3) Mobitz Type II 2nd degree or 3rd degree atrioventricular block or sick sinus syndrome, unless the patient had a pacemaker. 4) baseline QTC interval > $500 \mathrm{~ms}$. 5) treatment with Class la or Class III antiarrhythmic drugs. 6) history of macular edema, uveitis or diabetes mellitus, 7) elevated liver transaminase levels, 8) active infection, 9) known HIV, 10) known collagen vascular disease, or 11) concurrent use of immunosuppressive medications (other than dexamethasone for cerebral edema).

Study interventions: Fingolimod (Gilenya) is a sphingosine 1-phosphate receptor modulator that has been commercially available since 2010. A dose of $0.5 \mathrm{mg}$ daily is FDA approved for the treatment of patients with multiple sclerosis to reduce the frequency of clinical exacerbations. The major risks associated with fingolimod include first-dose bradycardia, macular edema, elevation of liver enzymes, increased risk of infections, and potential fetal harm. The baseline assessment of patients on this study was modeled 
after the standard recommendations for patients with multiple sclerosis. The assessment included a complete blood count, liver transaminase levels, an electrocardiogram and an ophthalmologic examination. Abnormalities in these assessments would preclude patients from participating in the study. To minimize risk, careful cardiac monitoring with the first dose of fingolimod was performed due to the possibility of first-dose bradycardia. Patients were observed for bradycardia for at least 6 hours after the first dose with hourly pulse and blood pressure measurements. Electrocardiograms (ECG) were obtained prior to dosing and at the end of the observation period. Patients who developed a heart rate $<45 \mathrm{bpm}$, new onset 2nd degree or higher atrioventricular block, lowest postdose heart rate at the end of the observation period, or symptomatic bradycardia were to be admitted overnight for continuous ECG monitoring and treatment, if necessary. As outlined in the fingolimod package insert, if treatment was discontinued for more than 1 day during weeks 1-2, or for more than 7 days during weeks 3-4, or for more than 14 days for weeks 5 and beyond, the same precautions for first dose monitoring were repeated. Fingolimod was administered daily for the first two weeks, then on Monday, Wednesday and Friday until the end of radiotherapy to minimize dosing interruptions. A detailed ophthalmologic exam (including dilated fundoscopy and optical coherence tomography) to identify macular edema was performed before starting fingolimod and after the seven weeks of treatment. Patients were advised to continue effective contraceptive practices for at least two months after discontinuing the fingolimod. Patients were allowed to receive standard steroid therapy for the treatment of their peritumoral edema. Pill counts were conducted weekly to ensure that $>80 \%$ of the prescribed fingolimod was taken. Fingolimod was purchased from Novartis for this study. Monitoring for infectious diseases, visual symptoms and blood counts to follow circulating lymphocyte levels were conducted on weekly visits during the radiation therapy and then every two months for one year or until any toxicities resolved.

Sample size: A total of 5 evaluable patients were to be enrolled on this study. Patients were considered evaluable if they received at least $80 \%$ of fingolimod doses throughout the duration of radiation or if they had significant toxicities. We estimated that at least $80 \%$ of accrued patients would be evaluable for the primary endpoint. Unevaluable patients could be replaced to complete the primary analysis. If, at any time, one patient experienced greater than or equal to Grade III infection attributable to fingolimodinduced lymphopenia within four months of starting fingolimod, we would conclude that radiation with temozolomide and fingolimod is not safe.

\section{Results - Human Study}

A total of five patients were accrued. All patients received over $80 \%$ of the prescribed fingolimod doses and were evaluable for the primary endpoint of the study. The characteristics of the enrolled patients are presented in Table 1. The median age of the patients was 37 years (range 23-69 years), Three of the five patients $(60 \%)$ were male, four $(80 \%)$ had glioblastoma, three (60\%) were IDH wildtype, and two of 4 patients $(50 \%)$ with a determined MGMT status were methylated. None of the patients were taking oral corticosteroids at baseline. All patients completed standard $60 \mathrm{~Gy}$ radiation with concurrent and adjuvant temozolomide. The median overall survival of these patients was 25 months (range 17-53+ months). As 
expected, patients with younger age, MGMT methylation, and IDH mutations had the most favorable survival outcomes.

Each of the five patients enrolled on this study had a baseline total lymphocyte count greater than 1000 and CD4 count greater than 400. As shown in Figure 3, each patient's total lymphocyte and CD4 count fell dramatically during the one week of fingolimod therapy that was administered before radiation was initiated. Their median total lymphocyte count prior to fingolimod was 1866 (range 1600-2040) and one week later the median was 512 (range 300-720). Total lymphocyte and CD4 counts remained very low ( $80 \%$ of patients had grade III-IV lymphopenia, defined as TLC $<500$ and/or CD 4 count $<200$ ) during entire six weeks of radiation and fingolimod. Although one of five $(20 \%)$ had recovery of the lymphocyte counts following the completion of radiation and fingolimod, in general both the total lymphocyte and CD4 counts remained low during the follow-up period as has previously been documented for patients with high grade gliomas treated with concurrent radiation and temozolomide. No grade III-IV opportunistic infections were observed either during this treatment or during the follow-up that lasted for a maximum of 1 year. In addition, there were no serious side effects noted secondary to fingolimod.

\section{Discussion}

Treatment-related lymphopenia is an under-appreciated toxicity of radiation therapy that likely results from the inadvertent radiation of circulating lymphocytes as they pass through the radiation field. Severe treatment-related lymphopenia is associated with inferior outcomes for patients with glioblastomas and other solid tumors.[1-6] It also appears to reduce the efficacy of immunologic interventions, such as vaccines or checkpoint inhibitors.[19-22] As a result, it is important to consider preventive strategies and approaches to reduce its severity.

Under normal conditions, lymphocytes circulate through the periphery and regularly pass through the lymphatic system, specifically lymph nodes (LNs), as they survey for evidence of pathogens. These lymphocytes require sphingosine-1-phosphate (S1P) signaling through its cognate receptor (S1PR1) in order to exit the lymph node. As such, this signaling pathway is an attractive target for modulating autoimmune diseases, such as multiple sclerosis, by interfering with those lymphocytes ability to exit the $\mathrm{LN}$ and cause pathogenesis. Alternatively, it stands to reason that this approach could be beneficial for protecting the circulating and highly radiosensitive lymphocytes from the off-target effects of radiotherapy by intentionally sequestering them in the $L N$ for the duration of treatment. Putatively, binding to S1PR1 on lymphocytes leads to internalization of the receptor, which thereby prevents the S1P/S1PR1 signaling required for lymphocyte egress from the LN. It is this proposed mechanism that results in a $70 \%$ decrease in circulating lymphocytes after one week of fingolimod administration. This is a reversible process, as lymphocytes will re-upregulate expression of S1PR1 once fingolimod treatment is completed. Even though patients on this drug have dramatically reduced circulating lymphocyte counts, they do not have a marked increase in opportunistic infections probably because their lymphocytes are redistributed but remain functional and within the body. In our animal study we have shown that the addition of fingolimod to radiation dramatically reduced circulating lymphocyte counts compared to 
cranial radiation alone. However, this severe lymphopenia did not result in worsened weight loss, performance measures, infections, or other obvious toxicities despite the additive reduction in lymphocyte counts. Perhaps more importantly, the addition of fingolimod did not significantly delay the post-radiation recovery in lymphocyte counts that is relatively rapid in murine models. It is important to note that the absolute cell counts are only a single facet of a potential effect of this treatment. It remains to be determined if sequestering these lymphocytes during radiotherapy alters their ability to mount an improved anti-tumor response.

In the human pilot safety study reported here, fingolimod resulted in a severe drop in total lymphocyte and CD4 counts when administered prior to initiation of radiation therapy and temozolomide as expected. The combined treatment with radiation, temozolomide and fingolimod resulted in striking lymphopenia, but without evident opportunistic infections or other toxicities. Lymphocyte counts were slow to recover once fingolimod was discontinued. This failure to rapidly repopulate lymphocyte counts could occur for a myriad of reasons. These patients continued to receive adjuvant temozolomide (a potent lympholytic drug) for 6 months in the adjuvant setting. In addition, they have been shown to have low IL-7 levels, which is a driver for lymphopoiesis; this observation has yet to be fully understood.[23] This is unlike what is detected in mice, where animals undergoing radiotherapy have an inverse relationship between lymphocyte counts and IL-7 levels in the LN, and the lymphocyte levels return to baseline in $~ 40$ days.

These two small pilot studies are the first to study the feasibility and safety of combining fingolimod with radiation in mice and humans. Taken together, these results provide important safety information suggesting that this novel and previously unexplored approach to reducing the number of circulating lymphocytes exposed to radiation deserves further study given the importance of the host immune system on cancer survival and response to immunologic interventions.

\section{Declarations}

Acknowledgment: Peter Calabresi donated fingolimod for the animal studies reported in this manuscript

Funding: National Cancer Institute: P30CA006973 Nelson (PI)

Conflicts of Interests: The authors have no conflicts of interest to declare that are relevant to the content of this article.

Availability of data and material: All data generated or analyzed during this study are included in this submitted manuscript.

Code availability: Not applicable

\section{Authors' contributions:}

Design and conduct of trial: AFP, DCJ, TRN, EV, JW, CGD, XY, SAG 
Authorship: AFP, DCJ, TRN, EV, JW, CGD, XY, SAG

Ethics approval: Animal experiments were approved by the Johns Hopkins' Institutional Animal Care and Use Committee. All procedures performed in the studies involving human participants were in accordance with the ethical standards of the institutional and/or national research committee and with the 1964 Helsinki Declaration and its later amendments or comparable ethical standards. The study was approved by the Institutional Review Board (IRB) of the Johns Hopkins University.

Consent to participate: Informed consent was obtained from all individual participants included in the stud

Consent for publication: Human data is de-identified and patients were informed that the study results would be considered for publication

\section{References}

1. Grossman SA, Ye X, Lesser G, et al. (2011) Immunosuppression in patients with high-grade gliomas treated with radiation and temozolomide. Clin Cancer Res.17(16):5473-5480.

2. Balmanoukian A, Ye X, Herman J, et al. (2012) The association between treatment-related lymphopenia and survival in newly diagnosed patients with resected adenocarcinoma of the pancreas. Cancer Invest. 30(8):571-576.

3. Campian JL, Sarai G, Ye X, Marur S, Grossman SA. (2014) Association between severe treatmentrelated lymphopenia and progression-free survival in patients with newly diagnosed squamous cell head and neck cancer. Head Neck. 36(12):1747-1753.

4. Campian JL, Ye X, Brock M, Grossman SA. (2013) Incidence, severity, and etiology of treatmentrelated lymphopenia in patients with stage III non-small cell lung cancer. Cancer Invest. 31:183-188.

5. Wild AT, Ye X, Ellsworth SG, et al. (2015) The association between chemoradiation-related lymphopenia and clinical outcomes in patients with locally advanced pancreatic adenocarcinoma. Am J Clin Oncol. 38(3):259-265.

6. Grossman SA, Ellsworth S, Campian J, et al. (2015) Survival in patients with severe lymphopenia following treatment with radiation and chemotherapy for newly diagnosed solid tumors. J Natl Compr Canc Netw. 13(10):1225-1231.

7. Yovino S, Grossman SA. (2012) Severity, etiology and possible consequences of treatment-related lymphopenia in patients with newly diagnosed high-grade gliomas. CNS Oncol. 1(2):149-154.

8. Yovino S, Kleinberg L, Grossman SA, et al. (2013) The etiology of treatment-related lymphopenia in patients with malignant gliomas: modeling radiation dose to circulating lymphocytes explains clinical observations and suggests methods of modifying the impact of radiation on immune cells. Cancer Invest. 31(2):140-144. 
9. Piotrowski AF, Nirschl TR, Velarde E, et al. (2018) Systemic depletion of lymphocytes following focal radiation to the brain in a murine model. Oncoimmunology. 7(7):e1445951.

10. Cronkite EP, Jansen CR, Mather GC, et al. Studies on lymphocytes. (1962) I. Lymphopenia produced by prolonged extracorporeal irradiation of circulating blood. Blood. 20:203-213.

11. Weeke E. (1973) The development of lymphopenia in uremic patients undergoing extracorporeal irradiation of the blood with portable beta units. Radiat Res. 56(3):554-559.

12. Ellsworth SG. (2018) Field size effects on the risk and severity of treatment-induced lymphopenia in patients undergoing radiation therapy for solid tumors. Adv Radiat Oncol. 3(4):512-519.

13. Durante M, Brauer-Krisch E, Hill M. Faster and safer? (2018) FLASH ultra-high dose rate in radiotherapy. Br J Radiol:;91(1082):20170628.

14. Wild AT, Herman JM, Dholakia AS, et al. (2016) Lymphocyte-Sparing Effect of Stereotactic Body Radiation Therapy in Patients With Unresectable Pancreatic Cancer. Int J Radiat Oncol Biol Phys. 94(3):571-579.

15. Shiraishi Y, Fang P, Xu C, et al. (2018) Severe lymphopenia during neoadjuvant chemoradiation for esophageal cancer: A propensity matched analysis of the relative risk of proton versus photon-based radiation therapy. Radiother Oncol.;128(1):154-160.

16. Byun HK, Kim N, Yoon HI, et al. (2019) Clinical predictors of radiation-induced lymphopenia in patients receiving chemoradiation for glioblastoma: clinical usefulness of intensity-modulated radiotherapy in the immuno-oncology era. Radiat Oncol. 14:51.

17. Francis G, Kappos L, O'Connor P, et al. (2014) Temporal profile of lymphocyte counts and relationship with infections with fingolimod therapy. Mult Scler. 20(4):471-480.

18. Nunamaker EA, Artwohl JE, (2013) Anderson RJ, Fortman JD. Endpoint refinement for total body irradiation of C57BL/6 mice. Comp Med 63:22-28

19. Diehl A, Yarchoan M, Hopkins A, Jaffee E, Grossman SA. (2017) Relationships between lymphocyte counts and treatment-related toxicities and clinical responses in patients with solid tumors treated with PD-1 checkpoint inhibitors. Oncotarget. 8(69):114268-114280.

20. Strowd RE, Russell G, Hsu F-C, et al. (2018) Immunogenicity of high-dose influenza vaccination in patients with primary central nervous system malignancy. Neurooncol Pract. 5(3): 176-183.

21. Ho WJ, Yarchoan M, Hopkins A, et al. (2018) Association between pretreatment lymphocyte count and response to PD1 inhibitors in head and neck squamous cell carcinomas. J Immunother Cancer. 6: 84.

22. Cho Y, Park S, Byun HK, et al. (2019) Impact of treatment-related lymphopenia on immunotherapy for advanced non-small cell lung cancer. Int J Radiat Oncol Biol Phys. 105 (5): 1065-1073.

23. Ellsworth S, Balmanoukian A, Kos F, et al. (2014) Sustained CD4+ cell driven lymphopenia without a compensatory IL7/II15 response among high-grade glioma patients treated with radiation and temozolomide. Oncoimmunology 3:e27357. 


\section{Tables}

\section{Table 1: Patient Characteristics}

\begin{tabular}{|c|c|c|c|c|c|c|c|}
\hline Patient & $\begin{array}{l}\text { Age } \\
\text { (years) }\end{array}$ & Histology & $\begin{array}{l}\text { Baseline } \\
\text { total lymph } \\
\text { count }\end{array}$ & $\begin{array}{l}\text { Baseline } \\
\text { CD4 } \\
\text { count }\end{array}$ & $\begin{array}{l}\text { IDH } \\
\text { status }\end{array}$ & $\begin{array}{l}\text { MGMT } \\
\text { Status }\end{array}$ & $\begin{array}{l}\text { Survival } \\
\text { (months) }\end{array}$ \\
\hline$\# 1$ & 65 & Glioblastoma & 1920 & 984 & $\begin{array}{l}\text { Wild } \\
\text { type }\end{array}$ & $\begin{array}{l}\text { Not } \\
\text { determined }\end{array}$ & 16 \\
\hline \#2 & 39 & Glioblastoma & 1810 & 1088 & $\begin{array}{l}\text { Wild } \\
\text { type }\end{array}$ & Unmethylated & 17 \\
\hline \#3 & 69 & Glioblastoma & 1960 & 1269 & $\begin{array}{l}\text { Wild } \\
\text { type }\end{array}$ & Methylated & 25 \\
\hline$\# 4$ & 23 & $\begin{array}{l}\text { Anaplastic } \\
\text { Astrocytoma }\end{array}$ & 2040 & 1043 & Mutant & Unmethylated & $42+$ \\
\hline \#5 & 37 & Glioblastoma & 1600 & 901 & Mutant & Methylated & $53+$ \\
\hline
\end{tabular}

\section{Figures}
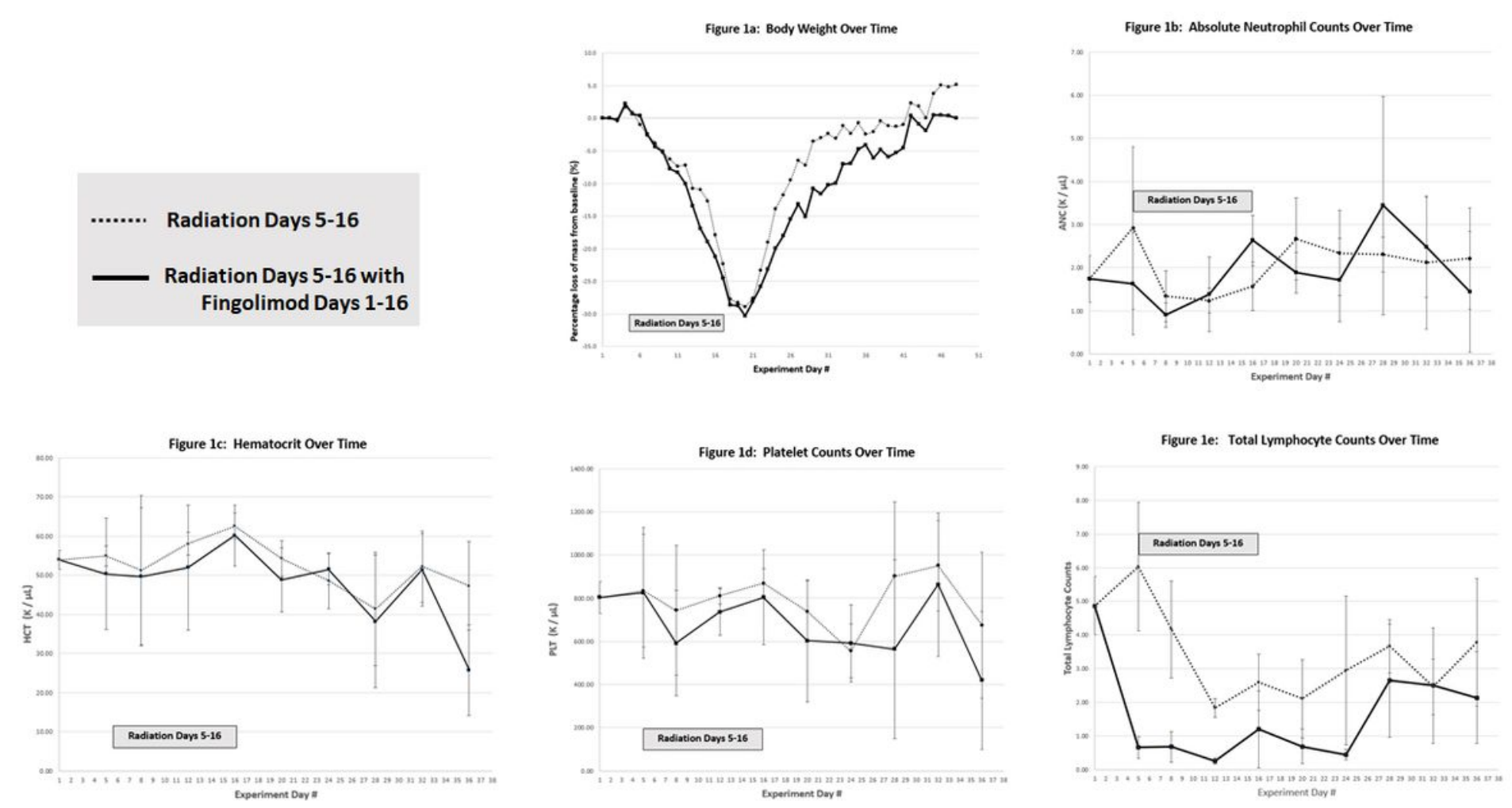

Figure 1 
Combining Fingolimod with Brain Irradiation in Mice Radiation causes transient weight loss followed by recovery to baseline in the murine model. There is no difference in the percent loss or return to normal weight in the mice treated with fingolimod in addition to radiation (Figure 1a). Adding fingolimod to radiation also does not substantially affect rodent absolute neutrophil counts (Figure 1b), hematocrit (Figure 1c), or platelet counts (Figure 1d). However, as illustrated in Figure 1e, pre-treating the animals with fingolimod results in a dramatic fall in circulating lymphocyte counts before radiation is initiated. These counts remain low during radiation and once the radiation and fingolimod are discontinued the lymphocyte counts recover similarly in animals treated with radiation alone and with radiation with temozolomide.

Patient population: $\quad \geq 18$ years, KPS $\geq 60$, newly diagnosed high grade astrocytoma, no contra-indications to fingolimod Study Design: $\quad$ Fingolimod for one week prior and during the 42 days of radiation and temozolomide Primary Objective: To determine if fingolimod can be safely combined with standard radiation and temolozolomide Secondary Objective: To obtain preliminary information regarding the ability of fingolimod to reduce radiationrelated lymphopenia at 3 months

Primary Endpoint: Grade III-IV toxicities and infections within 4 months of starting fingolimod Secondary Endpoint: Total lymphocyte counts 3 month after stopping fingolimod

Treatment Schema
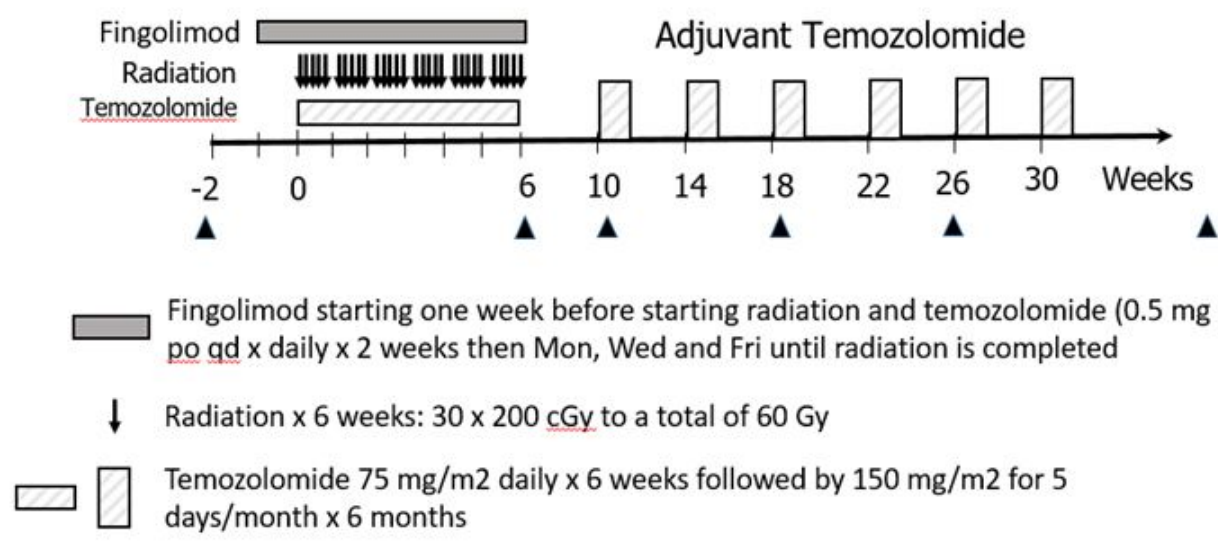

A Research blood collections at weeks $-2,6,10,18,26,46$

\section{Figure 2}

Safety Study of Fingolimod with Radiation and Temozolomide in Patients with Newly Diagnosed High Grade Gliomas 

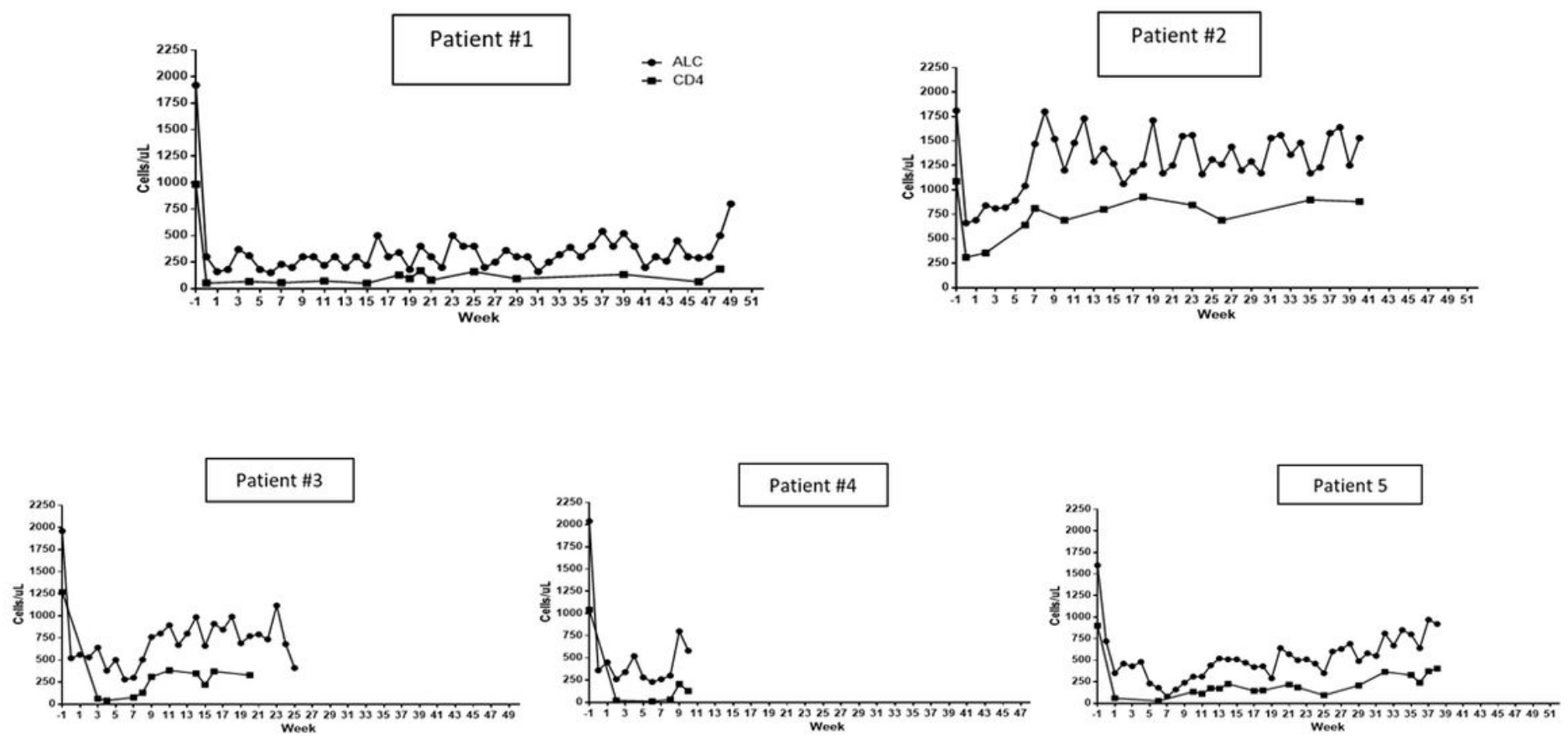

\section{Figure 3}

Serial absolute lymphocyte (ALC) and CD4 counts in patients treated with fingolimod for one week prior to the initiation of radiation and then daily during the six weeks of radiation. 